\title{
Thermo-optic noise in coated mirrors for high-precision optical measurements
}

\author{
M. Evans, ${ }^{1}$ S. Ballmer, ${ }^{2}$ M. Fejer, ${ }^{3}$ P. Fritschel, ${ }^{1}$ G. Harry, ${ }^{1}$ and G. Ogin ${ }^{2}$ \\ ${ }^{1}$ Massachusetts Institute of Technology, Cambridge, Massachusetts 02139, USA \\ ${ }^{2}$ California Institute of Technology, Pasadena, California 91125, USA \\ ${ }^{3}$ Stanford University, Stanford, California 94305, USA \\ (Received 25 July 2008; published 10 November 2008)
}

\begin{abstract}
Thermal fluctuations in the coatings used to make high reflectors are becoming significant noise sources in precision optical measurements and are particularly relevant to advanced gravitational-wave detectors. There are two recognized sources of coating thermal noise; mechanical loss and thermal dissipation. Thermal dissipation causes thermal fluctuations in the coating which produce noise via the thermoelastic and thermorefractive mechanisms. We treat these mechanisms coherently, give a correction for finite coating thickness, and evaluate the implications for Advanced LIGO.
\end{abstract}

DOI: 10.1103/PhysRevD.78.102003

PACS numbers: 04.80.Cc, 04.30.Db, 04.80.Nn

\section{INTRODUCTION AND MAIN RESULT}

Thermal fluctuations in the coatings used to make high reflectors are becoming significant noise sources in precision optical measurements [1-3]. Though masked by other noise sources in first generation interferometric gravitational-wave antennas (e.g., GEO, LIGO, TAMA, Virgo), designers of second generation gravitational-wave antennas expect coating thermal noise to be the dominant noise source in the detector's most sensitive frequency band [4]. Reduction of coating thermal noises has the potential to significantly increase the sensitivity, and thus the detection rate, of these large scale detectors.

Coating thermal noises are defined by differences between the coating material and the substrate material [5]. There are two recognized sources of coating thermal noise; mechanical loss and thermal dissipation. The first of these leads to "coating Brownian" noise, which, while not the topic of this paper, serves as measure against which we will compare our results [6]. The second, thermal dissipation in the coating, leads to temperature fluctuations, which can cause variation in the apparent position of an optic via thermal expansion of the coating, and thermal change in refractive index of the coating material, collectively known as "thermo-optic" noise [7].

Despite their common origin, coating thermoelastic noise (resulting from thermal expansion of the coating) and thermorefractive noise have not been treated in a coherent way $[8,9]$. Since the two mechanisms have the same order of magnitude, a coherent treatment has the potential to greatly change the predicted magnitude of their sum; thermo-optic noise.

The purpose of this paper is to unify the thermo-optic mechanisms. The formulaic result of this unification is presented later in this section, and derived in Sec. II. A correction for coatings of non-negligible thickness is given in III. In Sec. IV, we evaluate the thermo-optic and Brownian noises expected to be present in Advanced LIGO, given current understanding of coating material parameters and detector design. Finally, in the appendices we give equations for evaluating the average material constants of a multilayer coating, we derive the dependence of the reflection phase of a coating on its temperature, and we relate coating thermoelastic noise to substrate thermoelastic noise.

The power spectrum of coating thermal fluctuations responsible for thermo-optic noise, as observed by a sensing beam with a Gaussian profile, is given by [9]

$$
S_{\mathrm{TO}}^{\Delta T}=\frac{2 \sqrt{2}}{\pi} \frac{k_{B} T^{2}}{r_{G}^{2} \sqrt{\kappa C \omega}}
$$

(see Table I for a list of symbols, their definitions and units) [10].

These thermal fluctuations produce fluctuations in the phase of the field reflected by the mirror. This is equivalent to a change in the sensed position of the mirror, $\Delta z$, via the simple relation [11]

TABLE I. The physical constants, material parameters, and frequently used symbols in this paper.

\begin{tabular}{lcc}
\hline \hline Symbol & Name & SI unit \\
\hline$k_{B}$ & Boltzmann's constant & $J / K$ \\
$T$ & Mean temperature & $K$ \\
$\omega$ & Angular frequency & $\mathrm{rad} / \mathrm{s}$ \\
$C$ & Heat capacity per volume & $\mathrm{J} / \mathrm{Km}$ \\
$\kappa$ & Thermal conductivity & $\mathrm{W} / \mathrm{mK}$ \\
$n$ & Refractive index & \\
$\alpha$ & Thermal expansion & $1 / K$ \\
$\beta$ & $\partial n / \partial T$ & \\
$E$ & Young's Modulus & $N / \mathrm{m}^{2}$ \\
$\sigma$ & Poisson ratio & \\
$\lambda$ & Beam wavelength & $\mathrm{m}$ \\
$r_{G}$ & Beam radius (1/ $e^{2}$ power) & $\mathrm{m}$ \\
$d$ & Coating thickness & $\mathrm{m}$ \\
$\partial_{\phi}^{z}$ & $\partial \Delta z / \partial \phi=-\lambda / 4 \pi$ & $\mathrm{m}$ \\
\hline \hline
\end{tabular}




$$
\frac{\partial \Delta z}{\partial T}=\frac{\partial \Delta z}{\partial \phi} \frac{\partial \phi}{\partial T}=\frac{-\lambda}{4 \pi} \frac{\partial \phi}{\partial T}=\partial_{\phi}^{z} \frac{\partial \phi}{\partial T},
$$

where we define $\partial_{\phi}^{z} \equiv-\lambda / 4 \pi$ to avoid repetition.

We combine thermoelastic and thermorefractive (TE and TR) mechanisms to write the total sensitivity to temperature of the coating reflection phase as

$$
\partial_{\phi}^{z} \frac{\partial \phi_{c}}{\partial T} \simeq \bar{\alpha}_{c} d-\bar{\beta} \lambda,
$$

where $\bar{\alpha}_{c}$ is the effective coefficient of thermal expansion of the coating and $\bar{\beta}$ is the effective thermorefractive coefficient (defined in Appendices A and B). The derivations presented in the following sections lead us to express the total thermo-optic noise spectrum as

$$
S_{\mathrm{TO}}^{\Delta z} \simeq S_{\mathrm{TO}}^{\Delta T}\left(\bar{\alpha}_{c} d-\bar{\beta} \lambda-\bar{\alpha}_{s} d \frac{C_{c}}{C_{s}}\right)^{2} .
$$

Though this paper includes some further refinements to previous works, Eq. (4) contains the primary result: the relative sign between the TE and TR mechanisms is negative. This relative sign can lead to cancellation and a reduction in thermo-optic noise, some implications of which are discussed further in Sec. IV.

\section{A. Symbol definitions}

The physical constants, material parameters, and frequently used symbols in this paper are given in Table I. Material parameters that appear with a subscript refer to either the bulk substrate material parameter, subscript $s$, the average coating parameter, subscript $c$, or to one of the coating materials $L$, for low-refractive index, or $H$, for high-refractive index.

Material parameters which appear without a subscript, but as a function of $\vec{r}$, take on the value of the material at the location $\vec{r}$. Thus, $\bar{\alpha}(\vec{r})$ is $\bar{\alpha}_{c}$ when $\vec{r}$ describes a point in the coating, and $\bar{\alpha}_{s}$ for points in the substrate.

Bars are used above symbols to express an "effective" coefficient. These coefficients have the same units as their barless counterparts and the same general meaning, though taken in a specific context. For example, $\bar{\alpha}$ has the same units as $\alpha$, and is a thermal expansion coefficient, but only in the context of a semi-infinite medium.

As a notational convention, $S_{Y}^{X}$ means "the power spectrum of fluctuations in $X$ due to noise mechanism $Y$." The units of such a spectrum are the units of $X^{2} / \mathrm{Hz}$.

\section{REFLECTION PHASE NOISE}

This section will derive Eq. (4) from the fluctuationdissipation theorem (FDT) [12]. We start by deriving Fejer's result [13] for thermoelastic noise using Levin's simpler approach [9]. The solution to the more general problem of thermo-optic noise is then found following the same path.
The thermal fluctuations which are the source of thermooptic noise are important to optical measurements because they change the result of position measurements based on reflecting a field from a mirror. The fields used in these measurements are well described by a normalized Gaussian intensity profile [14]

$$
I\left(r_{\perp}\right)=\frac{2}{\pi r_{G}^{2}} e^{-2 r_{\perp}^{2} / r_{G}^{2}}
$$

where $r_{G}$ is the beam radius, and $r_{\perp}^{2}=x^{2}+y^{2}$ is the radius perpendicular to the beam's propagation direction (along the $z$ axis).

To go from thermal fluctuations to measured displacement noise we return to the foundation of this analysis. Our application of the FDT starts with a gedanken experiment in which we consider an oscillating power injection in a small volume $\delta V$ located at $\vec{r}$ :

$$
\frac{P}{\delta V}=T F_{0} \sin (\omega t) q(\vec{r}),
$$

where $F_{0}$ is an arbitrary scale factor with SI units of $\mathrm{m}^{2} \times$ $\mathrm{W} / \mathrm{m}^{3}=\mathrm{N} / \mathrm{s}$, and $\omega$ is the frequency of interest [15]. The form factor $q(\vec{r})$ connects the measurement variable $\hat{z}$ to temperature fluctuations $\delta T(\vec{r}, t)$ in the mirror via

$$
\hat{z}=\oint \delta T(\vec{r}, t) q(\vec{r}),
$$

where the integral is formally over all space, though the integrand is presumably zero outside the mirror and its coating.

Power injection leads to heat flow and thus dissipation as expressed by

$$
W=\left\langle\oint \frac{\kappa}{T}(\vec{\nabla} \delta T)^{2}\right\rangle,
$$

where the average $\langle\cdots\rangle$ is over cycles of the power injection. Finally, the FDT relates this dissipation to the spectral density of noise in the associated measurement variable by

$$
S^{\Delta z}=\frac{8 k_{B} T W}{F_{0}^{2}} .
$$

In the next section, as an illustrative example, we will derive Fejer's result for thermoelastic noise using the approach outlined above. The same approach is applied to the more complicated problem of thermo-optic noise in Sec. II B.

\section{A. An example: Coating thermoelastic noise}

As a concrete example, we will first apply the above formalism to derive coating thermoelastic noise in the absence of any thermorefractive mechanism, (previously performed in $[8,13])$. The solution to the nontrivial problem of thermal expansion of a thin coating on a semiinfinite substrate is presented in [13], appears in our Eq. (A2), and is contained in the effective thermal expansion 
coefficient $\bar{\alpha}$. Under the assumption that the coating elastic coefficients are similar to those of the substrate material, the effective thermal expansion coefficient simplifies to

$$
\bar{\alpha}_{c} \sim 2 \alpha_{c}\left(1+\sigma_{c}\right) .
$$

The same expression is valid for the constrained thermal expansion of the bulk material in a semi-infinite substrate, $\bar{\alpha}_{s}$.

Referring back to Eq. (7), the thermoelastic readout variable

$$
\hat{z}_{\mathrm{TE}}=\oint \delta T(\vec{r}, t) q_{\mathrm{TE}}(\vec{r})
$$

describes the sensing beam's averaging of the thermally induced displacement of points on the mirror's surface. From this we can write simply

$$
q_{\mathrm{TE}}(\vec{r})=I\left(r_{\perp}\right) \bar{\alpha}(\vec{r}),
$$

which leads to a thermoelastic power injection

$$
\frac{P_{\mathrm{TE}}}{\delta V}=T F_{0} \sin (\omega t) I\left(r_{\perp}\right) \bar{\alpha}(\vec{r}) .
$$

To remove the component of power injection which results in little temperature gradient and thus little heat flow, we subtract the substrate contribution

$$
\begin{aligned}
\frac{1}{C(\vec{r})} \frac{P_{\mathrm{TE}_{c}}}{\delta V} & =\frac{1}{C(\vec{r})} \frac{P_{\mathrm{TE}}}{\delta V}-\frac{1}{C_{s}} \frac{P_{\mathrm{TE}_{s}}}{\delta V} \\
& =T F_{0} \sin (\omega t) I\left(r_{\perp}\right)\left(\frac{\bar{\alpha}(\vec{r})}{C(\vec{r})}-\frac{\bar{\alpha}_{s}}{C_{s}}\right) .
\end{aligned}
$$

We can then recast this into the form of (6) as

$$
\frac{P_{\mathrm{TE}_{c}}}{\delta V}=T F_{0} \sin (\omega t) q_{\mathrm{TE}_{c}}(\vec{r}),
$$

where we have identified the coating thermoelastic readout form factor

$$
q_{\mathrm{TE}_{c}}(\vec{r})=I\left(r_{\perp}\right)\left(\bar{\alpha}(\vec{r})-\bar{\alpha}_{s} \frac{C(\vec{r})}{C_{s}}\right),
$$

which is zero in the substrate by design [16].

To maintain the simplicity of this example, we will assume that the coating and substrate are uniform, and that the coating is of thickness $d$ which is small with respect to the thermal diffusion length

$$
r_{T}=\sqrt{\frac{\kappa}{C \omega}} .
$$

With this assumption, we can consider all energy to be generated in this thin layer at the surface of the substrate and then flow inward. Integrating (14) over $z$ we compute the energy flux into the substrate to be

$$
\frac{P_{\mathrm{TE}_{c}}}{\delta A}=T F_{0} \sin (\omega t) I\left(r_{\perp}\right) \Delta \bar{\alpha} d,
$$

where

$$
\Delta \bar{\alpha}=\bar{\alpha}_{c}-\bar{\alpha}_{s} \frac{C_{c}}{C_{s}} .
$$

In order to connect this heat injection to $W$ in (8) we solve the diffusion equation

$$
C \frac{\partial \delta T}{\partial t}=\kappa \nabla^{2} \delta T
$$

with the boundary condition that the injected energy flows inward

$$
\frac{P_{\mathrm{TE}_{c}}}{\delta A}=-\left.\kappa_{s} \frac{\partial \delta T}{\partial z}\right|_{z=0},
$$

which ignores the very small radiation loss, as in [9].

Further assuming that $r_{G} \gg r_{T}$, we can ignore diffusion in the transverse dimensions, which yields the solution

$$
\frac{\partial \delta T}{\partial z} \simeq \frac{-T F_{0}}{\kappa_{s}} e^{\left(-z / \sqrt{2} r_{T}\right)} \sin \left(\omega t-\frac{z}{\sqrt{2} r_{T}}\right) I\left(r_{\perp}\right) \Delta \bar{\alpha} d,
$$

from which we can compute the power dissipation

$$
\begin{aligned}
W_{\mathrm{TE}_{c}} \simeq\left\langle\oint \frac{\kappa_{s}}{T}\left(\frac{\partial \delta T}{\partial z}\right)^{2}\right\rangle \\
W_{\mathrm{TE}_{c}} \simeq \frac{T F_{0}^{2}}{2 \sqrt{2} \pi r_{G}^{2} \kappa_{s}} r_{T}(\Delta \bar{\alpha} d)^{2} .
\end{aligned}
$$

Finally, returning to (9), we arrive at the coating thermoelastic noise spectrum

$$
S_{\mathrm{TE}_{c}}^{\Delta z}=\frac{2 \sqrt{2} k_{B} T^{2}}{\pi r_{G}^{2} \sqrt{\kappa_{s} C_{s} \omega}}(\Delta \bar{\alpha} d)^{2}=S_{\mathrm{TO}}^{\Delta T}(\Delta \bar{\alpha} d)^{2},
$$

which is equal to that of $[8,13]$ under their simplifying assumptions.

\section{B. Coating thermo-optic noise}

The thermoelastic noise described above assumes that the relevant readout variable is based on the position of the surface of the mirror. Interferometric sensors are, however, actually sensitive to the reflection phase of a surface as well as its position.

In the case of reflection from a planar surface, the position and reflection phase are related simply by $\delta z=$ $-\delta \phi \lambda / 4 \pi=\partial_{\phi}^{z} \delta \phi$, but for multilayer coatings the relationship can be more complicated. To account for this we generalize (11) to yield the thermo-optic readout variable,

$$
\hat{z}_{\mathrm{TO}}=\partial_{\phi}^{z} \oint \delta T(\vec{r}, t) I\left(r_{\perp}\right)\left(\frac{\partial \phi(z)}{\partial T}-\frac{C(z)}{C_{s}} \frac{\partial \phi_{s}}{\partial T}\right),
$$

where as before we have subtracted the substrate contribution so as to remove the component of heat injection which can be handled adiabatically. From this we identify the thermo-optic form factor 


$$
q_{\mathrm{TO}}(\vec{r})=I\left(r_{\perp}\right) \partial_{\phi}^{z}\left(\frac{\partial \phi(z)}{\partial T}-\frac{C(z)}{C_{s}} \frac{\partial \phi_{s}}{\partial T}\right),
$$

which is, as before, zero in the substrate.

Plugging into (6), we get

$$
\frac{P_{\mathrm{TO}}}{\delta V}=T F_{0} \sin (\omega t) I\left(r_{\perp}\right) \partial_{\phi}^{z}\left(\frac{\partial \phi(z)}{\partial T}-\frac{C(z)}{C_{s}} \frac{\partial \phi_{s}}{\partial T}\right) .
$$

Again we assume that the coating and substrate are uniform, and $d \ll r_{T} \ll r_{G}$, so we can integrate over $z$ to get the energy flux

$$
\frac{P_{\mathrm{TO}}}{\delta A}=T F_{0} \sin (\omega t) I\left(r_{\perp}\right)\left(\partial_{\phi}^{z} \frac{\partial \phi_{c}}{\partial T}-\bar{\alpha}_{s} d \frac{C_{c}}{C_{s}}\right),
$$

where

$$
\frac{\partial \phi_{c}}{\partial T}=\int_{0}^{d} d z \frac{\partial \phi(z)}{\partial T}
$$

is the overall reflection phase sensitivity of the coating to temperature, as described in Appendix B.

Following the path used for (23) above, we arrive at

$$
W_{\mathrm{TO}} \simeq \frac{T F_{0}^{2}}{2 \sqrt{2} \pi r_{G}^{2} \kappa_{s}} r_{T}\left(\partial_{\phi}^{z} \frac{\partial \phi_{c}}{\partial T}-\bar{\alpha}_{s} d \frac{C_{c}}{C_{s}}\right)^{2}
$$

and thus

$$
S_{\mathrm{TO}}^{\Delta z}=S_{\mathrm{TO}}^{\Delta T}\left(\partial_{\phi}^{z} \frac{\partial \phi_{c}}{\partial T}-\bar{\alpha}_{s} d \frac{C_{c}}{C_{s}}\right)^{2},
$$

which accounts for both thermo-optic mechanisms in $\partial \phi_{c} / \partial T$, the coating's overall reflection phase sensitivity to temperature. This can be combined with the approximate expression in Eq. (3) to arrive at our main result, Eq. (4). To obtain a more precise result, the thickness of the coating must be corrected for as described in Sec. III, and the value of $\partial \phi_{c} / \partial T$ computed as described in Appendix B.

\section{THICK COATING CORRECTION}

Here we will allow for finite thickness coatings by removing the assumption that $d \ll r_{T}$, while continuing to assume $r_{G} \gg r_{T}$ [17]. To do this we will need to solve the heat diffusion equation accounting for power deposition and diffusion in the coating. Generalizing (19) to include a source term, but limiting heat flow to the $z$ axis

$$
C \frac{\partial \delta T}{\partial t}=\kappa \frac{\partial^{2} \delta T}{\partial z^{2}}+\frac{P}{\delta L}
$$

with the one-dimensional power injection

$$
\frac{P_{\mathrm{TO}}}{\delta L}=\frac{1}{I\left(r_{\perp}\right)} \frac{P_{\mathrm{TO}}}{\delta V}=T F_{0} \sin (\omega t)\left(\partial_{\phi}^{z} \frac{\partial \phi(z)}{\partial T}-\bar{\alpha}_{s} \frac{C_{c}}{C_{s}}\right) .
$$

We will approximate the thermo-optic power deposition in the coating with a constant thermoelastic component, and a Dirac delta function for the thermorefractive component since its effect is limited to the first few layers of the coating. We can express this as

$$
\frac{P_{\mathrm{TO}}}{\delta L} \simeq T F_{0} \sin (\omega t)\left(\bar{\alpha}_{c}-\delta(z) \beta_{\mathrm{TR}}-\bar{\alpha}_{s} \frac{C_{c}}{C_{s}}\right),
$$

where we define

$$
\beta_{\mathrm{TR}}=\bar{\alpha}_{c} d-\partial_{\phi}^{z} \frac{\partial \phi_{c}}{\partial T} \simeq \bar{\beta} \lambda
$$

with $\partial \phi_{c} / \partial T$ and $\bar{\beta}$ as given in Appendix B.

Following the method used in [13], we transform (30) to a second-order differential equation in $z$ :

$$
\theta(z)-\frac{1}{\gamma^{2}} \frac{\partial^{2} \theta(z)}{\partial z^{2}}=-\rho(z),
$$

where the relationships between the new and old variables are

$$
\begin{aligned}
\delta T(z, t) & =\mathcal{R} e\left(e^{i \omega t} \theta(z)\right) \\
\frac{P(z, t)}{\delta L} & =\omega C \mathcal{R} e\left(-i e^{i \omega t} \rho(z)\right) \\
\gamma & =\sqrt{i \frac{\omega C}{\kappa}}=\frac{\sqrt{i}}{r_{T}} .
\end{aligned}
$$

The homogeneous solutions to this equation in the coating and substrate are

$$
\theta_{h c}(z)=\theta_{d c} \cosh \left(\gamma_{c} z\right) \quad \theta_{h s}(z)=\theta_{d s} \exp \left(-\gamma_{s} z\right),
$$

where the coefficients $\theta_{d}$ will be determined by boundary conditions at $z=d$. These equations satisfy the boundary conditions of no heat flow at $z=0$ or $z=\infty$.

The particular solutions needed are for the two kinds of sources, TE and TR, both of which are limited to the coating. The source terms are

$$
\begin{aligned}
\rho_{\mathrm{TE}} & =\frac{T F_{0}}{\omega C_{c}}\left(\bar{\alpha}_{c}-\bar{\alpha}_{s} \frac{C_{c}}{C_{s}}\right)=\frac{T F_{0}}{\omega C_{c}} \Delta \bar{\alpha} \\
\rho_{\mathrm{TR}}(z) & =-\delta(z) \frac{T F_{0}}{\omega C_{c}} \beta_{\mathrm{TR}}=\delta(z) \bar{\rho}_{\mathrm{TR}}
\end{aligned}
$$

and the corresponding particular solutions are

$$
\theta_{p \mathrm{TE}}=-\rho_{\mathrm{TE}} \quad \theta_{p \mathrm{TR}}(z)=-\gamma_{c} \bar{\rho}_{\mathrm{TR}} \exp \left(-\gamma_{c} z\right) .
$$

We put all this together with boundary conditions at $z=d$ that ensure continuity of temperature and conservation of energy

$$
\begin{aligned}
\theta_{h s}(d) & =\theta_{p \mathrm{TE}}+\theta_{p \mathrm{TR}}(d)+\theta_{h c}(d) \\
\kappa_{s} \frac{\partial}{\partial z} \theta_{h s}(d) & =\kappa_{c} \frac{\partial}{\partial z}\left(\theta_{p \mathrm{TR}}(d)+\theta_{h c}(d)\right)
\end{aligned}
$$

to find 


$$
\begin{aligned}
\theta_{d c} & =\rho_{\mathrm{TE}}+\bar{\rho}_{\mathrm{TR}} \gamma_{c} \exp \left(-\gamma_{c} d\right)(1-R) / \psi_{d} \\
\theta_{d s} & =-R \exp \left(\gamma_{s} d\right)\left(\rho_{\mathrm{TE}} \sinh \left(\gamma_{c} d\right)+\bar{\rho}_{\mathrm{TR}} \gamma_{c}\right) / \psi_{d} \\
\psi_{d} & =\cosh \left(\gamma_{c} d\right)+R \sinh \left(\gamma_{c} d\right) \\
R & =\sqrt{\frac{\kappa_{c} C_{c}}{\kappa_{s} C_{s}}}=\frac{\kappa_{c} \gamma_{c}}{\kappa_{s} \gamma_{s}}=\frac{\kappa_{c} r_{T s}}{\kappa_{s} r_{T c}} .
\end{aligned}
$$

Before we lose ourselves among the equations, recall that our goal is to find the time averaged dissipation $W$, which is related to the temperature gradient in Eq. (8). We now have $\theta(z)$ in hand, and (35) relates this to $\delta T$, so our destination is near. Summing the homogeneous and particular solutions to get $\theta(z)$, and taking the derivative with respect to $z$, we find

$$
\begin{aligned}
& \frac{\partial \theta_{c}(z)}{\partial z}=\gamma_{c}\left(\theta_{d c} \sinh \left(\gamma_{c} z\right)+\gamma_{c} \bar{\rho}_{\mathrm{TR}} \exp \left(-\gamma_{c} z\right)\right) \\
& \frac{\partial \theta_{s}(z)}{\partial z}=-\gamma_{s} \theta_{d s} \exp \left(-\gamma_{s} z\right) .
\end{aligned}
$$

From Eqs. (8) and (35) we can see that

$$
\begin{aligned}
W_{\mathrm{TO}}^{\text {thick }} & \simeq \frac{1}{\pi r_{G}^{2}}\left\langle\int_{0}^{\infty} d z \frac{\kappa}{T}\left(\frac{\partial \delta T}{\partial z}\right)^{2}\right\rangle \\
& \simeq \frac{1}{2 \pi r_{G}^{2}} \int_{0}^{\infty} d z \frac{\kappa}{T}\left|\frac{\partial \theta(z)}{\partial z}\right|^{2},
\end{aligned}
$$

where the transverse integrals over $I\left(r_{\perp}\right)^{2}$ have already been performed.

To arrive at a correction factor for thick coatings, we normalize the corrected thermo-optic dissipation above by that of a thin coating given in Eq. (28),

$$
\Gamma_{t c}=\frac{W_{\mathrm{TO}}^{\text {thick }}}{W_{\mathrm{TO}}}=\frac{S_{\mathrm{TO}}^{\Delta z_{\text {thick }}}}{S_{\mathrm{TO}}^{\Delta z}} .
$$

Taking the integral over the coating and substrate, we end with a complicated expression for the correction factor

$$
\begin{aligned}
\Gamma_{t c} & =\frac{p_{E}^{2} \Gamma_{0}+p_{E} p_{R} \xi \Gamma_{1}+p_{R}^{2} \xi^{2} \Gamma_{2}}{R \xi^{2} \Gamma_{D}} \\
\Gamma_{0} & =2(\sinh (\xi)-\sin (\xi))+2 R(\cosh (\xi)-\cos (\xi)) \\
\Gamma_{1} & =8 \sin (\xi / 2)(R \cosh (\xi / 2)+\sinh (\xi / 2)) \\
\Gamma_{2} & =\left(1+R^{2}\right) \sinh (\xi)+\left(1-R^{2}\right) \sin (\xi)+2 R \cosh (\xi) \\
\Gamma_{D} & =\left(1+R^{2}\right) \cosh (\xi)+\left(1-R^{2}\right) \cos (\xi)+2 R \sinh (\xi),
\end{aligned}
$$

where we have made the following substitutions

$$
p_{R}=\frac{\bar{\rho}_{\mathrm{TR}}}{d \rho_{\mathrm{TE}}+\bar{\rho}_{\mathrm{TR}}}, \quad p_{E}=\frac{d \rho_{\mathrm{TE}}}{d \rho_{\mathrm{TE}}+\bar{\rho}_{\mathrm{TR}}}
$$

using the dimensionless, frequency dependent, scale factor

$$
\xi=\frac{\sqrt{2} d}{r_{T c}}=\sqrt{\frac{2 \omega C_{c}}{\kappa_{c}}} d .
$$

Note that the power deposition fractions $p_{E}$ and $p_{R}$ can also be written as

$$
p_{R}=\frac{-\bar{\beta} \lambda}{\Delta \bar{\alpha} d-\bar{\beta} \lambda}, \quad p_{E}=\frac{\Delta \bar{\alpha} d}{\Delta \bar{\alpha} d-\bar{\beta} \lambda} .
$$

Applying this correction to Eq. (4) gives

$$
S_{\mathrm{TO}}^{\Delta z}=S_{\mathrm{TO}}^{\Delta T} \Gamma_{t c}(\Delta \bar{\alpha} d-\bar{\beta} \lambda)^{2} .
$$

For $d \ll r_{T c}$ or $\xi \ll 1$, we can use the much simpler expansion

$$
\Gamma_{t c} \simeq 1+\frac{p_{E}^{2}+3\left(p_{R}-R^{2}\right)}{3 R} \xi-\frac{p_{E}-3\left(1-R^{2}\right)}{6} \xi^{2},
$$

which goes to 1 as $\xi$ goes to 0 . In the case of a very thick coating, with $d \gg r_{T c}$, the thermal fluctuations which generate noise via TE and TR mechanisms become independent, and thus they add in quadrature [18]

$$
\Gamma_{t c} \simeq \frac{2 p_{E}^{2}}{R(1+R) \xi^{2}}+\frac{p_{R}^{2}}{R} .
$$

Thus, this correction expands our understanding beyond the simple notion that the TE and TR mechanisms have a relative negative sign. Now we can say that TE and TR mechanisms have a relative negative sign if $d \ll r_{T c}$, are partially coherent and partially canceling if $d \sim r_{T c}$, and act as independent noises if $d \gg r_{T c}$.

\section{IMPLICATIONS FOR ADVANCED LIGO}

Having clarified the relationship between the thermooptic mechanisms, a recomputation of the impact of this noise source is in order. We will also take this opportunity to use the most recent information about the physical properties of the materials involved, and to apply an additional correction factor for the less-than-infinite size of the mirror. To highlight the implications of this work, the results will be compared with Harry's result for coating Brownian noise [6].

The Advanced LIGO mirrors are high reflectors with a multilayer coating of alternating $\mathrm{SiO}_{2}$ and $\mathrm{Ta}_{2} \mathrm{O}_{5}$. The input mirrors will have a power transmission of $T=$ $1.4 \%$ with $r_{G}=5.5 \mathrm{~cm}$, while the end mirrors will have $T \simeq 5 \mathrm{ppm}$ with $r_{G}=6.2 \mathrm{~cm}$. The mirrors are made of fused silica, are $34 \mathrm{~cm}$ in diameter and $20 \mathrm{~cm}$ thick for a total mass of $40 \mathrm{~kg}$. In Figs. 1 and 2 we plot the coating related noises for coatings made of 1/4-wave doublets.

We take the finite test-mass correction from [19] which, with the mirror and beam-size parameters given above, is $C_{f s m} \simeq 0.98$. This multiplicative factor affects only the thermoelastic mechanism, 
ITM Coating Noise: $\mathrm{N}$ doublet $=8, \mathrm{~T}=1.4 \%$

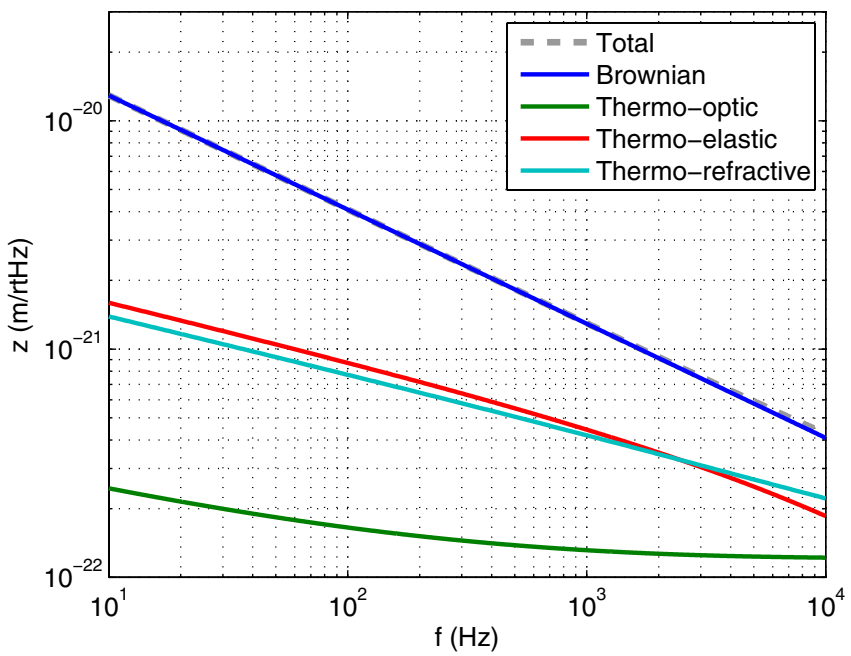

FIG. 1 (color online). Thermo-optic noises and Brownian noise for an Advanced LIGO input mirror.

$$
\Delta \bar{\alpha}_{f s m}=C_{f s m} \Delta \bar{\alpha}
$$

as it represents a bending of the optic due to strains produced by the coating. Adding this correction to Eq. (43) gives

$$
S_{\mathrm{TO}}^{\Delta z}=S_{\mathrm{TO}}^{\Delta T} \Gamma_{t c}\left(\Delta \bar{\alpha}_{f s m} d-\bar{\beta} \lambda\right)^{2}
$$

where we use $\Delta \bar{\alpha}_{f s m}$ in (42) when computing $\Gamma_{t c}$.

Figure 3 shows a representative Advanced LIGO sensitivity curve [20]. While the difference between the result of Eq. (47) and a conservative estimate which simply takes the sum of the TR and TE mechanisms is less than $10 \%$, our coherent treatment of TO noise makes clear that it

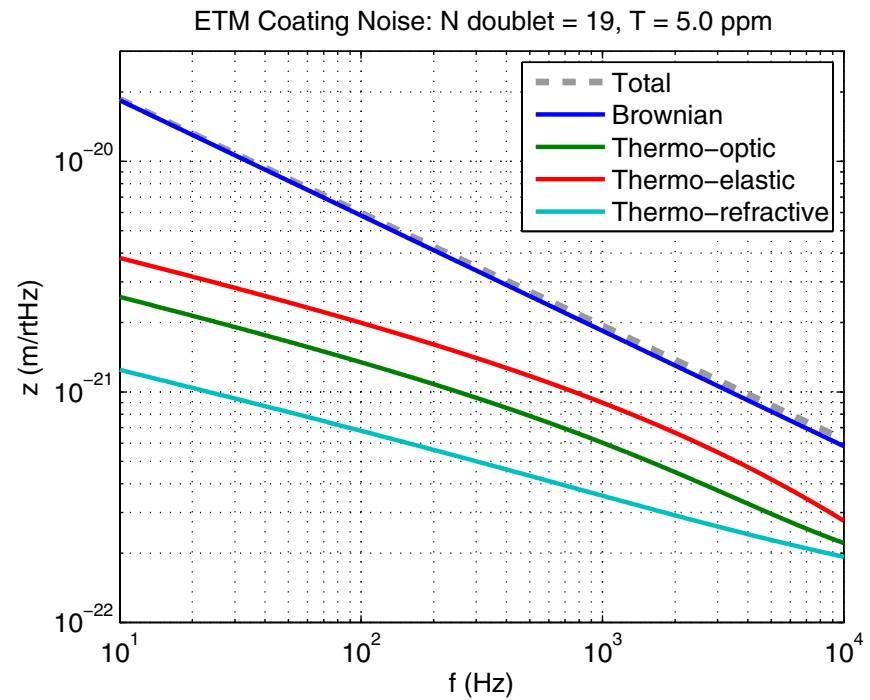

FIG. 2 (color online). Thermo-optic noises and Brownian noise for an Advanced LIGO end mirror.

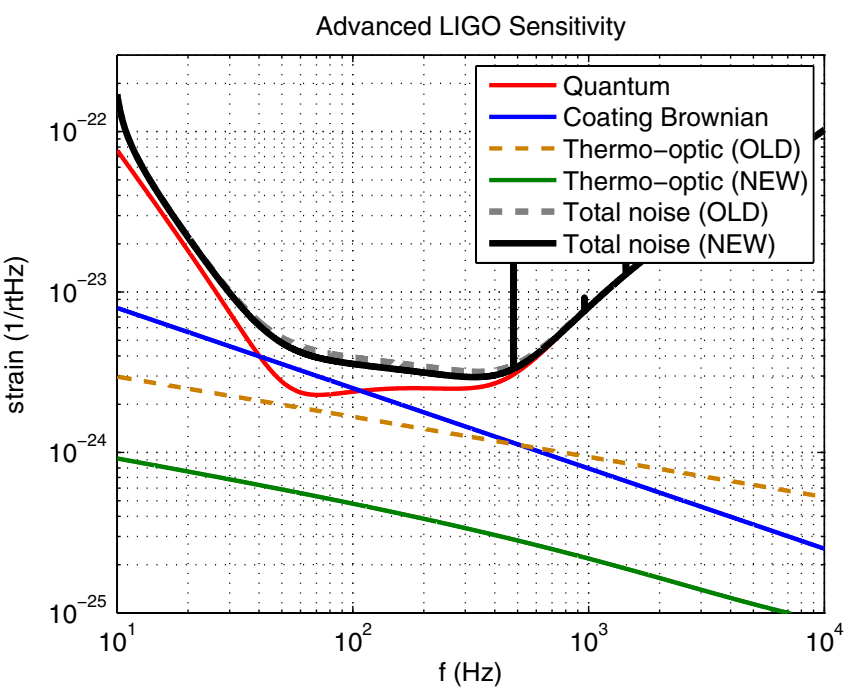

FIG. 3 (color online). An Advanced LIGO sensitivity curve. The thermo-optic curve labeled "NEW" uses Eq. (47), while the "OLD" curve uses a conservative estimate of TO noise: the sum of TR and TE, with the TE correction factor of 1.56 from [8].

should not be considered a driving force in Advanced LIGO coating design.

The material parameters used to make the figures in this section are given in Table II. It should be noted that some of these parameters are poorly constrained. The thermal conductivity of $\mathrm{Ta}_{2} \mathrm{O}_{5}$ is simply assumed to match that of sapphire [13]. Fortunately this only affects the thick coating correction factor, and no reasonable value significantly changes the result below $1 \mathrm{kHz}$. The value of $\beta$ for $\mathrm{Ta}_{2} \mathrm{O}_{5}$ is also poorly constrained, but again the range of tolerable values is large. Thermo-optic noise remains below the conservative "OLD" curve in Fig. 3 for values between

TABLE II. The values of material parameters used for Figs. 13. These values are taken from [13], with the exception of $\beta_{\mathrm{Ta}_{2} \mathrm{O}_{5}}$ noted in the text.

\begin{tabular}{lcc}
\hline \hline Symbol & $\mathrm{Ta}_{2} \mathrm{O}_{5}$ & Unit \\
\hline$\alpha$ & 3.6 & $10^{-6} / \mathrm{K}$ \\
$\beta$ & 14 & $10^{-6} / \mathrm{K}$ \\
$\kappa$ & 33 & $\mathrm{~W} / \mathrm{mK}^{3}$ \\
$C$ & 2.1 & $\mathrm{GJ} / \mathrm{Km}^{3}$ \\
$E$ & 140 & $\mathrm{GPa}$ \\
$\sigma$ & 0.23 & \\
$n_{H}$ & 2.06 & \\
\hline \hline \hline Symbol & & $\mathrm{Unit}$ \\
\hline$\alpha$ & $\mathrm{SiO}_{2}$ & $10^{-6} / \mathrm{K}$ \\
$\beta$ & 0.51 & $10^{-6} / \mathrm{K}$ \\
$\kappa$ & 8 & $\mathrm{~W} / \mathrm{mK}^{3}$ \\
$C$ & 1.38 & $\mathrm{GJ} / \mathrm{Km}{ }^{3}$ \\
$E$ & 1.64 & $\mathrm{GPa}$ \\
$\sigma$ & 72 & \\
$n_{L}$ & 0.17 & \\
\hline \hline
\end{tabular}


$-10^{-4} / \mathrm{K}$ and $3 \times 10^{-4} / \mathrm{K}$. The value of $\beta_{\mathrm{Ta}_{2} \mathrm{O}_{5}}$ used herein, from Gretarsson [19], is comparable to previous values $[21,22]$.

\section{CONCLUSION}

Thermo-optic noise results from thermal fluctuations in the coatings used to make high-reflection mirrors. These thermal fluctuations affect the measured position of a mirror through the thermoelastic and thermorefractive mechanisms. While both of these mechanisms have been known for some years, they were not treated coherently. The coherent treatment presented herein shows that these two mechanisms appear with a relative negative sign. The effect is to essentially reduce thermo-optic noise to the point of insignificance for second generation gravitationalwave antennas. While it is true that our current knowledge of the properties of coating materials is imprecise, it seems unlikely that better measurements, while desirable, will bring thermo-optic noise back into the realm of relevance. This fact should help to guide coating research in the coming years.

\section{APPENDIX A: COATING AVERAGE PROPERTIES}

Optical coatings are made from alternating layers of materials with different refractive indices. For properties other than the refractive index, as long as the length scales involved $\left(r_{T}\right.$ and $\left.r_{G}\right)$ are large compared to the layer thickness (typically $<\lambda / 2$ ), we can use suitably averaged material properties to represent the coating. The equations given in this section are all taken from [13], and are repeated here only for completeness and clarity.

The thermal expansion coefficient for a given layer $k$ in the coating is

$$
\bar{\alpha}_{k}=\alpha_{k} \frac{1+\sigma_{s}}{1-\sigma_{k}}\left[\frac{1+\sigma_{k}}{1+\sigma_{s}}+\left(1-2 \sigma_{s}\right) \frac{E_{k}}{E_{s}}\right]
$$

and the volume average coefficient for a coating with $N$ layers each of thickness $d_{k}$ is

$$
\bar{\alpha}_{c}=\sum_{k=1}^{N} \bar{\alpha}_{k} \frac{d_{k}}{d},
$$

where $d$ is the total coating thickness

$$
d=\sum_{k=1}^{N} d_{k}
$$

To compute the correction factor in Sec. III the average thermal properties of the coating are needed. The heat capacity is a simple volume average,

$$
C_{c}=\sum_{k=1}^{N} C_{k} \frac{d_{k}}{d}
$$

while the average thermal conductivity involves the in- verse,

$$
\kappa_{c}=\left(\sum_{k=1}^{N} \frac{1}{\kappa_{k}} \frac{d_{k}}{d}\right)^{-1}
$$

\section{APPENDIX B: REFLECTION PHASE OF A MULTILAYER COATING}

In this Appendix we describe the method we use for computing the reflection phase of a multilayer coating. The initial discussion is somewhat pedantic, but it serves to give us a consistent notation which we develop in the subsections detailing the thermoelastic and thermorefractive mechanisms.

We start with the effective reflectivity of the interface between materials with refractive indices $n_{1}$ and $n_{2}$, passing from material 1 to material 2,

$$
r_{1,2}=\frac{n_{1}-n_{2}}{n_{1}+n_{2}}
$$

Given two such transitions, from 1 to 2 and from 2 to 3 , we can equate the reflectivity to that of a two mirror cavity

$$
r_{1,2,3}=\frac{-r_{2,1}+r_{2,3} e^{-i \phi_{2}}}{1-r_{2,1} r_{2,3} e^{-i \phi_{2}}},
$$

where $\phi_{2}$ is the round-trip phase in material 2.

Note that the reflectivity of the 2 to 1 transition appears in (B2) with the indices in the order seen from inside the cavity. In the following text we will use the relation $r_{k+1, k}=-r_{k, k+1}$ to keep the indices in increasing order, and then drop the second index, such that $r_{k} \equiv r_{k, k+1}$.

If we number the interfaces in our coating in the order of increasing depth (i.e., the coating layer in contact with the vacuum is 1 , and the layer in contact with the substrate is $N$ ), we can define a recursion relation using (B2)

$$
\bar{r}_{k}=e^{-i \phi_{k}} \frac{r_{k}+\bar{r}_{k+1}}{1+r_{k} \bar{r}_{k+1}},
$$

where $\bar{r}_{k}=e^{-i \phi_{k}} r_{k, k+1, \ldots, N}$ is the effective reflectivity of a coating layer, including the round-trip in that layer. The base case for this recursion relation is the transition from the $N$ th coating layer to the substrate,

$$
\bar{r}_{N}=e^{-i \phi_{N}} r_{N, s},
$$

which can be evaluated with (B1).

Extending our coating to include the external vacuum as layer 0 provides a natural end to the recursion. The reflectivity of the coating is then given by $r_{c}=\bar{r}_{0}$, and we can use $\phi_{0}$ to account for the overall expansion of the coating into the vacuum with $\phi_{0}=\Delta_{c} / \partial_{\phi}^{z}$, where $\Delta_{c}$ is the total change in coating thickness.

To use (B3) to compute changes in reflection phase, one must take the derivative with respect to the round-trip phase in each layer. Here we give the recursion relation and base case for these derivatives, 


$$
\frac{\partial \bar{r}_{k}}{\partial \phi_{j}}= \begin{cases}e^{-i \phi_{k}} \frac{1-r_{k}^{2}}{\left(1+r_{k} \bar{r}_{k+1}\right)^{2}} \frac{\partial \bar{r}_{k+1}}{\partial \phi_{j}} & k<j \\ -i \bar{r}_{k} & k=j \\ 0 & k>j .\end{cases}
$$

From the derivatives of the reflectivity of each layer, the derivative of the reflection phase of the coating as a whole is

$$
\frac{\partial \phi_{c}}{\partial \phi_{k}}=\frac{\partial \arg \left(\bar{r}_{0}\right)}{\partial \phi_{k}}=\operatorname{Im}\left(\frac{1}{\bar{r}_{0}} \frac{\partial \bar{r}_{0}}{\partial \phi_{k}}\right) .
$$

For any quarter or half-wave coating, $\bar{r}_{0}$ is entirely real and its phase derivatives are entirely imaginary, so much of the apparent complexity is not real.

\section{Thermally induced changes}

For phase changes induced by a uniform change in temperature we have

$$
\frac{\partial \phi_{c}}{\partial T}=\sum_{k=0}^{N} \frac{\partial \phi_{c}}{\partial \phi_{k}} \frac{\partial \phi_{k}}{\partial T} .
$$

The phase change due to thermoelastic and thermorefractive effects in a coating with layers of thickness $d_{k}$ are

$$
\begin{aligned}
& \frac{\partial \phi_{k}}{\partial T}=\frac{4 \pi}{\lambda}\left(\beta_{k}+\bar{\alpha}_{k} n_{k}\right) d_{k}=\frac{4 \pi}{\lambda} B_{k} d_{k} \\
& \frac{\partial \phi_{0}}{\partial T}=-\frac{4 \pi}{\lambda} \sum_{k=1}^{N} \bar{\alpha}_{k} d_{k}=\bar{\alpha}_{c} \frac{d}{\partial_{\phi}^{z}},
\end{aligned}
$$

where, as previously noted, we use $\phi_{0}$ to account for the overall expansion of the coating. For any real coating, one can evaluate this expression numerically, and thus find $\partial \phi_{c} / \partial T$ for that coating.

\section{Relative sign of TE and TR in 1/4-wave coatings}

Of particular interest are high-reflection coatings made of 1/4-wave layers of alternating low- $n$ and high- $n$ material. For simplicity, we will assume that the high- $n$ layers have $n_{H}>n_{s}$ and that the low- $n$ layers have $n_{L}=n_{s}$. Thus, the reflectivity from high $n$ to low $n$ is

$$
r_{H}=\frac{n_{H}-n_{L}}{n_{L}+n_{H}} .
$$

As a transition from the vacuum, the first layer is of low- $n$ material and $1 / 2$ wave in optical thickness, such that

$$
r_{0}=\frac{1-n_{L}}{1+n_{L}} .
$$

Summarizing, these coatings have the following properties:

$$
e^{-i \phi_{k}}=\left\{\begin{array}{ll}
1 & k \& l t ;=1 \\
-1 & k>1
\end{array} \quad r_{k}= \begin{cases}r_{0} & k=0 \\
r_{H} & k \text { even } \\
-r_{H} & k \text { odd }\end{cases}\right.
$$

From the above we can at least determine the signs of the various phase derivatives. We start by noting that

$$
\operatorname{sign}\left(\bar{r}_{k}\right)= \begin{cases}-1 & k=1 \\ -1 & k \text { even } \\ 1 & k \text { odd }\end{cases}
$$

and that (B5) inverts the sign of the derivative for each layer with $k>1$. Even numbered layers start with $\partial \bar{r}_{k} / \partial \phi_{k}$ positive, experience $k-2$ sign inversions, and thus end with a positive sign. Odd numbered layers, on the other hand, start with $\partial \bar{r}_{k} / \partial \phi_{k}$ negative, experience an odd number of sign inversions, and thus these also end with a positive sign. Since $\bar{r}_{0}$ is negative, we are ensured that

$$
\operatorname{sign}\left(\frac{\partial \phi_{c}}{\partial \phi_{k}}\right)=-1, \quad \text { for all } k \text {. }
$$

It follows that, for any high-reflection coating of this construction, thermoelastic and thermorefractive effects will appear with opposite sign in (B7), thanks to the relative minus sign in (B8).

\section{Approximation for high reflectors}

While Eqs. (B7) and (B8) are accurate and easy to use in numerical computation, they offer little intuitive understanding and fail to provide a concise expression for the thermo-optic mechanisms. To address this, we give an approximation which is useful for high-reflection coatings.

The thermoelastic mechanism, which arises from motion of the coating's surface, is accounted for by the $k=0$ term in Eq. (B7) [also the second line in Eq. (B8)]. This term can be expressed in terms of the average coating expansion coefficient as

$$
\frac{\partial z_{\mathrm{TE}}}{\partial T}=\partial_{\phi}^{z} \frac{\partial \phi_{0}}{\partial T}=\bar{\alpha}_{c} d .
$$

The thermorefractive mechanism is accounted for by the terms with $k>0$ in (B7), which can be thought of as the change in reflection phase as measured at a point on the coating's surface. Both $\partial n / \partial T$ and thermal expansion play a role in changing the reflection phase of a coating, so while this mechanism is refereed to as "thermorefractive," this is something of a misnomer. We define an effective TR coefficient $\bar{\beta}$ such that [23]

$$
\frac{\partial z_{\mathrm{TR}}}{\partial T}=-\bar{\beta} \lambda .
$$

For a coating made entirely of $1 / 4$-wave doublets, $\bar{\beta}$ can be approximated by

$$
\bar{\beta}_{\mathrm{QW}} \simeq \frac{n_{L}^{2} B_{H}+n_{H}^{2} B_{L}}{4\left(n_{H}^{2}-n_{L}^{2}\right)},
$$

where $B_{X}$ is the fractional change in optical path length with respect to temperature in material $X$ :

$$
B_{X}=\beta_{X}+\bar{\alpha}_{X} n_{X},
$$




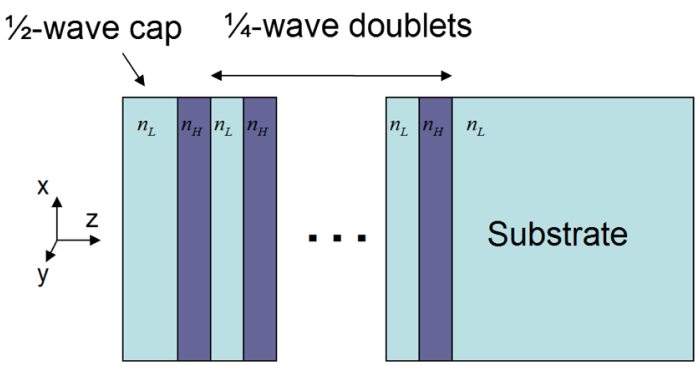

FIG. 4 (color online). A high-reflection coating made of $1 / 4$-wave doublets with a $1 / 2$-wave cap layer.

with $X \in\{L, H\}$ either the low-index material $L$ or the high-index material $H$, as given in [7]. A 1/4-wave cap layer is, however, counterproductive and not used in high reflectors.

To find $\bar{\beta}$ for the common high reflection coating (made of $1 / 4$-wave doublets with a $1 / 2$-wave cap layer, as shown in Fig. 4), we modify $\bar{\beta}_{\mathrm{QW}}$ by approximating $\bar{r}_{k} \simeq \operatorname{sign}\left(\bar{r}_{k}\right)$. Since the sign of $\bar{r}_{0}$ is minus in the 1/4-wave case and plus in the $1 / 2$-wave case, each term in (B5) with $j>0$ is reduced by

$$
\frac{\left(1+r_{0}\right)^{2}}{\left(1-r_{0}\right)^{2}}=\frac{1}{n_{L}^{2}}
$$

Furthermore, we must include the additional $1 / 4$ wave of material in the thicker cap layer

$$
\frac{\partial \phi_{c, \mathrm{HW}}}{\partial \phi_{1}}=\frac{1+r_{0}}{1-r_{0}}=\frac{1}{n_{L}}
$$

so that the additional temperature sensitivity is

$$
\bar{\beta}_{L} \frac{\partial \phi_{c, \mathrm{HW}}}{\partial \phi_{1}} \frac{1}{4 n_{L}}=\bar{\beta}_{L} \frac{1}{4 n_{L}^{2}} .
$$

Putting these corrections together gives

$$
\bar{\beta} \simeq \frac{\bar{\beta}_{\mathrm{QW}}}{n_{L}^{2}}+\frac{B_{L}}{4 n_{L}^{2}}
$$

which can be rearranged to

$$
\bar{\beta} \simeq \frac{B_{H}+B_{L}\left(2\left(n_{H} / n_{L}\right)^{2}-1\right)}{4\left(n_{H}^{2}-n_{L}^{2}\right)} .
$$

Returning to the motive for this exploration, Eq. (3) arises simply from the sum of TE and TR terms:

$$
\partial_{\phi}^{z} \frac{\partial \phi_{c}}{\partial T}=\frac{\partial z_{\mathrm{TE}}}{\partial T}+\frac{\partial z_{\mathrm{TR}}}{\partial T} \simeq \bar{\alpha}_{c} d-\bar{\beta} \lambda .
$$

For alternating layers of $\mathrm{SiO}_{2}$ and $\mathrm{Ta}_{2} \mathrm{O}_{5}$, this approximation is within a few percent for coatings with more than $\sim 6$ doublets.

\section{APPENDIX C: RELATIONSHIP TO SUBSTRATE THERMOELASTIC NOISE}

The spectrum of thermal fluctuations described by (1), and derived previously in [8,9], can be rearranged with the help of the thermal diffusion length. If we rewrite (1) as

$$
S_{\mathrm{TO}}^{\Delta T}=\frac{\sqrt{2} k_{B} T^{2}}{\omega C_{s} r_{T}^{3}} \frac{2 r_{T}^{2}}{\pi r_{G}^{2}},
$$

we can see that the first fraction is the spectral density of the thermodynamic fluctuation in a volume defined by the diffusion length, while the second is the Gaussian beam average over these volumes.

The coating thermo-optic coupling is designed such that a similar equation applied to the substrate would result in zero. The reason for this is that the loss associated with the coating results from nonadiabatic heat flow due to the difference between the coating and substrate. The substrate thermoelastic noise, on the other hand, results from adiabatic heat flow on the scale of the beam radius $r_{G}$, and is thus smaller by a factor of $\sim r_{T} / r_{G}$. See, for instance, Eq. (2) in [8] which can be written in our notation as

$$
S_{\mathrm{TE}_{s}}^{\Delta z}=\frac{4 k_{B} T^{2}}{\sqrt{\pi} \omega C_{s} r_{G}^{3}}\left(\bar{\alpha}_{s} r_{T}\right)^{2}=S_{\mathrm{TO}}^{\Delta T} \sqrt{2 \pi} \frac{r_{T}}{r_{G}}\left(\bar{\alpha}_{s} r_{T}\right)^{2} .
$$

To give an idea of the relative importance of substrate and coating thermoelastic noise, we divide the coating thermoelastic noise in (23) by $(\mathrm{C} 1)$ and define the thermoelastic ratio

$$
R_{\mathrm{TE}} \equiv \frac{S_{\mathrm{TE}_{c}}^{\Delta z}}{S_{\mathrm{TE}_{s}}^{\Delta z}}=\frac{d^{2} r_{G}}{\sqrt{2 \pi} r_{T}^{3}} \frac{\Delta \bar{\alpha}^{2}}{\bar{\alpha}_{s}^{2}} .
$$

In the case of a gravitational-wave interferometer we have roughly $r_{G} \sim 5 \mathrm{~cm}$ and $d \sim 5 \mu \mathrm{m}$. For a fused-silica substrate, $r_{T} \sim 40 \mu \mathrm{m}$ around $100 \mathrm{~Hz}$, such that $R_{\mathrm{TE}} \sim$ $10\left(\Delta \bar{\alpha} / \bar{\alpha}_{s}\right)^{2} \sim 150$, indicating that the substrate contribution is insignificant. For a sapphire substrate $\left(r_{T} \sim\right.$ $130 \mu \mathrm{m}$ and $\alpha_{s}=5.6 \times 10^{-6}$ ), on the other hand, the substrate contribution is dominant $R_{\mathrm{TE}} \sim 0.2\left(\Delta \bar{\alpha} / \bar{\alpha}_{s}\right)^{2} \sim$ 0.1 . 
[1] K. Numata, A. Kemery, and J. Camp, Phys. Rev. Lett. 93, 250602 (2004).

[2] A. D. Ludlow, X. Huang, M. Notcutt, T. Zanon-Willette, S. M. Foreman, M. M. Boyd, S. Blatt, and J. Ye, Opt. Lett. 32, 641 (2007).

[3] F. Marquardt, A.A. Clerk, and S.M. Girvin, arXiv:0803.1164v1; Geo web site, http://www.geo600.de; Ligo web site, http://www.ligo.caltech.edu; Tama web site, http://tamago.mtk.nao.ac.jp; Virgo web site, http:// www.virgo.infn.it.

[4] P. Fritschel, Proceedings of SPIE Vol. 4856 "Gravitational Wave Detection", edited by Mike Cruise and Peter Saulson (SPIE, Bellingham, WA, 2003), pp. 282-291; http://arxiv.org/gr-qc/0308090.

[5] To be precise, coating Brownian noise is simply the coating's contribution to the total Brownian noise, which is worth discussing separately because the coating materials typically have much higher loss than the substrate. Coating thermo-optic noise, on the other hand, is a phenomenon which results from the differences between the coating and the substrate.

[6] G. M. Harry et al., Classical Quantum Gravity 24, 405 (2007).

[7] V. B. Braginsky, M. L. Gorodetsky, and S. P. Vyatchanin, Phys. Lett. A 271, 303 (2000).

[8] V. B. Braginsky and S. P. Vyatchanin, Phys. Lett. A 312, 244 (2003).

[9] Y. Levin, arXiv:0710.2710v1.

[10] In all uses of $S_{\mathrm{TO}}^{\Delta T}$ herein, the material parameters refer to those of the substrate.

[11] We use plane-wave propagation with phase evolution given by $E(x, t)=E(0,0) \exp \left(i \omega_{0} t-i\left(2 \pi d_{\text {prop }} / \lambda+\right.\right.$ $\phi)$ ), where $\omega_{0}=2 \pi c / \lambda, c$ is the speed of light, $t$ is the time of the measurement, $d_{\text {prop }}$ is the distance propagated, and $\phi$ is an additional phase due to propagation delay. A mirror displacement $\Delta z$ shortens the propagation distance of the reflected field, and thus changes $d_{\text {prop }}$ by $-2 \Delta z$, while a change in reflection phase contributes directly to $\phi$. Note that the mirror position $\Delta z$ is defined such that an expansion of the mirror coating results in a positive mirror position

[12] H. B. Callen and T. A. Welton, Phys. Rev. 83, 34 (1951).
[13] M. M. Fejer, S. Rowan, G. Cagnoli, D. R. M. Crooks, A. Gretarsson, G. M. Harry, J. Hough, S. D. Penn, P. H. Sneddon, and S. P. Vyatchanin, Phys. Rev. D 70, 082003 (2004).

[14] The definition of the Gaussian beam radius used by $[8,9,24]$ is a factor of $\sqrt{2}$ different from the definition used in this paper and elsewhere [13,25]. This results in an additional factor of 2 in the numerator of the noise spectra equations with respect to $[8,9,24]$.

[15] Previous authors start from pressure injection [13,24,26]. Pressure is converted to strain in the material, and then to power injection via the thermoelastic mechanism. We essentially follow the path of Levin [21], who speaks of entropy injection, which is equivalent to energy injection as expressed in his Eq. (12). The application of a derivative with respect to time converts energy injection to power injection, a well-defined quantity even in the presence of diffusion.

[16] $q_{\mathrm{TE}_{c}}(\vec{r})$ is zero in the substrate simply because, for any value of $\vec{r}$ in the substrate $\bar{\alpha}(\vec{r})=\bar{\alpha}_{s}$ and $C(\vec{r})=C_{s}$. The reason for this choice of form factor is explained in Appendix C.

[17] To give some round numbers for gravitational-wave interferometers, $r_{G} \sim 5 \mathrm{~cm}$ and $d<10 \mu \mathrm{m}$. For a $\mathrm{SiO}_{2}$ substrate, $r_{T} \sim 40 \mu \mathrm{m}$ around $100 \mathrm{~Hz}$.

[18] Removing the thermorefractive component (i.e., setting $p_{E}=1, p_{R}=0$ ), and normalizing by their 0th order coefficient $R \xi / 2$, gives the results found in [13].

[19] A. Gretarsson (private communication).

[20] To avoid clutter, many of the noise sources which form the "Total" curve are not shown in Fig. 3.

[21] W. H. Cheng, S. F. Chi, and A. K. Chu, Thin Solid Films 347, 233 (1999).

[22] Chuen-LinTien, C.-C. Jiang, C.-C. Lee, and K.-P. Chuang, J. Mod. Opt. 47, 1681 (2000).

[23] We chose to make $\bar{\beta}$ positive and write explicitly the negative sign shown in the previous section.

[24] Y. T. Liu and K. S. Thorne, Phys. Rev. D 62, 122002 (2000).

[25] A.E. Siegman, Lasers (University Science Books, Sausalito, CA, 1986).

[26] Y. Levin, Phys. Rev. D 57, 659 (1998). 